\title{
The effect of Lipo prostaglandin E1 on cauda equina blood flow in patients with lumbar spinal canal stenosis: myeloscopic observation
}

\author{
K Yone*,1, T Sakou ${ }^{1}$ and Y Kawauchi ${ }^{1}$ \\ ${ }^{1}$ Department of Orthopaedic Surgery, Faculty of Medicine, Kagoshima University, Japan
}

\begin{abstract}
Study Design: Myeloscopic examination was performed to observe the cauda equina in patients with lumbar spinal canal stenosis before and after treatment with Lipo prostaglandin E1, a strong peripheral vasodilator.

Objectives: The purpose of this study was to clarify the effects of Lipo prostaglandin E1 on blood flow in the cauda equina in patients with lumbar spinal canal stenosis.

Setting: Japan, Kagoshima

Methods: We performed myeloscopic observations of morphological changes in blood vessels running along the cauda equina in 11 patients with lumbar spinal canal stenosis before and after treatment with Lipo prostaglandin E1.

Results: In six of these patients, dilation of the running blood vessels was observed immediately after administration. In all of the patients who exhibited a dilation of vessels on the surface of the cauda equina, intermittent claudication and lower extremity pain and/or numbness lessened immediately after examination. However, none of the patients who exhibited no morphological changes in the vessels along the cauda equina after administration of Lipo prostaglandin E1 experienced any improvement of symptoms at the time of examination.

Conclusion: Results of this study suggest that Lipo prostaglandin E1 may enhance blood flow in the cauda equina and improve clinical symptoms in some patients with lumbar spinal stenosis.
\end{abstract}

Sponsorship: None

Keywords: myeloscopy; lumbar spinal canal stenosis; Lipo prostaglandin E1; cauda equina; intermittent claudication

\section{Introduction}

As the elderly population has increased, the number of patients with lumbar spinal canal stenosis complaining of lower back pain, lower extremity pain and/or numbness, and intermittent claudication has tended to increase year by year. Since the concept of this disease was established by Verbiest in $1954,{ }^{1}$ many studies of its etiology and diagnostic and therapeutic methods have been performed. ${ }^{2-8}$ However, the pathogenesis of this disease's symptoms, especially intermittent claudication, remains obscure. Two theories have been developed regarding the cause of these symptoms. The first is that the development of functional disorder of the spinal cord, cauda equina and nerve root is due to mechanical compression. The second theory is that, in addition to mechanical compression, circulatory failure in the spinal cord, cauda equina and nerve root also plays a role in the development of symptoms. In the treatment of this disease, conservative therapy such as physical therapy,

*Correspondence: Dr K Yone, Department of Orthopaedic Surgery, Faculty of Medicine, Kagoshima University, 8-35-1 Sakuragaoka, Kagoshima, Japan 890 medication with a non-steroid analgesic drug, muscle relaxants and vitamins, epidural nerve block, and nerve root blocks are the first choice. When improvement is not obtained with these conservative treatments, patients undergo surgery, and decompression of nerve tissues such as the dural tube and nerve root is performed. However, patients with this disease are usually of advanced age, and surgical therapy is often not performed because of the possibility of complications, and in many cases only conservative therapy is applicable.

Recently, several studies ${ }^{9,10}$ on the improvement of clinical symptoms of this disease by treatment with prostaglandin E1 (PGE1) have been reported in Japan. PGE1 has strong peripheral vasodilating activity and platelet aggregation-inhibiting activity, and its usefulness has been confirmed both for the treatment of chronic arterial obstruction and other ischemic diseases and for the maintenance of blood circulation after blood vessel reconstruction. ${ }^{11,12}$ However, it is not yet clear whether PGE1 improves blood circulation in the cauda equina and nerve root of patients with lumbar spinal canal stenosis. 
The purpose of this study was to clarify the effects of PGE1 on blood flow in the cauda equina by myeloscopic observation of the morphological changes in the blood vessels running along the cauda equina in patients with lumbar spinal canal stenosis before and after treatment with PGE1.

\section{Subjects and methods}

The subjects were 11 patients with lumbar spinal canal stenosis, including seven males and four females. Age at the time of examination ranged from $45-78$ years (mean, 63.6 years), and duration of symptoms ranged from 4 months to 5 years (mean, 2 years). Intermittent claudication and lower extremity pain and/or numbness were found in all of the patients, and low back pain was found in ten patients. The femoral nerve stretch (FNS) test was positive in all of the patients, and straight leg raising (SLR) test was positive in three patients. Sensory abnormalities of the inferior limbs were found in seven patients, and weakness of the lower extremities was found in eight patients. Spinal canal stenosis was found in every subject on myelography and MRI; the regions affected were L23, L3-4, and L4-5 in one patient, L3-4 and L4-5 in four patients, L3-4, L4-5, and L5-S in two patients, L4-5 in two patients, and L4-5 and L5-S1 in two patients. Informed consent was obtained from all patients.

The devices and procedures are described briefly in this article and are as reported previously. ${ }^{13} \mathrm{~A}$ rigid endoscope $1.7 \mathrm{~mm}$ in diameter (Olympus Optical Co., Tokyo) was used for myeloscopy. Patients lay on their side on the operating table. Under local anesthesia, a trocar $2 \mathrm{~mm}$ in diameter was inserted into the subdural space through a $3-4 \mathrm{~mm}$ long skin incision over the lumbar interspinous space. An endoscope was then inserted inside the dura mater through the trocar. The endoscope was connected to a television monitor and a video set by a specific cable for observation and recording. The insertion level was that maximally stenosed as observed on myelograms or MRI. When insertion at a maximally stenotic level was impossible, an adjacent level was used. The levels observed were L3-4 in five patients and L4-5 in six patients.

Lipo PGE1, an emulsified PGE1 (Alprostagil, Taisyo Co., Tokyo), was dispersed in physiological saline $(10 \mu \mathrm{g}$ in $10 \mathrm{ml})$ and slowly injected into a vein over $5 \mathrm{~min}$. This preparation is not instantaneously inactivated in the body, but it efficiently accumulates in lesions after intravenous injection and releases prostaglandin. ${ }^{14}$

Myeloscopic observation was performed from $5 \mathrm{~min}$ before administration of PGE1 until $20 \mathrm{~min}$ after administration. Morphological changes in the vessels running along the cauda equina and adhesive changes in the cauda equina were observed and were recorded on videotape. These recorded findings were evaluated by all authors and one anatomist not participating in this study. Ooi et $a l^{6}$ observed adhesive arachnoiditis in cauda equina using myeloscopy, and classified the severity of adhesive changes in three degrees, slight, moderate and marked, based on findings for the dura matter, arachnoid and cauda equina (Table 1). In this study, the appearance of adhesive arachnoiditis was classified using Ooi's classification.

\section{Results}

Adhesive arachnoiditis was observed in all of the patients, and was slight in three, moderate in six, and marked in two (Table 2). In 6 of 11 patients $(54.5 \%)$, dilation of the blood vessels running along the cauda equina was observed within $5 \mathrm{~min}$ after intravenous injection of Lipo PGE1.

Immediately after the examination, all of the patients who exhibited dilation of vessels on the surface of the cauda equina had improved ability to walk and decrease in numbness and/or pain in lower extremities to various degrees, with complete disappearance of these symptoms in one patient and partial improvements in five patients, while none of these patients obtained alleviation of lumbago. The FNS test was improved in four of six patients, and the SLR test was improved in one of two patients who had a positive SLR test before examination. Sensory abnormalities of the lower extremities were slightly improved in one of three patients who exhibited sensory disturbance before examination, and weak-

Table 1 Ooi's classification of adhesive arachnoiditis ${ }^{6}$

\begin{tabular}{ll}
\hline Slight & Thickening of dura mater \\
& Opaque or cloudy arachnoid \\
Cauda equina is almost normal & Findings of dura mater and arachnoid \\
are same as 'slight' & Loss of glossiness and/or discoloration \\
of cauda equina & Hyperaemia of the vessels on the surface \\
of cauda equina & Findings of dura mater and arachnoid \\
are same as 'slight' & Redundant or aggregated cauda equina \\
\hline
\end{tabular}

Table 2 Patient data

\begin{tabular}{lccccl}
\hline $\begin{array}{l}\text { Patient } \\
\text { No. }\end{array}$ & $\begin{array}{c}\text { Age } \\
\text { (yrs) }\end{array}$ & Sex & $\begin{array}{c}\text { Level of } \\
\text { examination }\end{array}$ & $\begin{array}{c}\text { Dilation of } \\
\text { vessels }\end{array}$ & $\begin{array}{l}\text { Ooi's } \\
\text { classification }\end{array}$ \\
\hline 1 & 66 & M & L3-4 & + & Marked \\
2 & 65 & M & L3-4 & + & Moderate \\
3 & 74 & F & L4-5 & + & Moderate \\
4 & 78 & M & L3-4 & - & Moderate \\
5 & 59 & M & L4-5 & + & Moderate \\
6 & 51 & F & L4-5 & + & Moderate \\
7 & 55 & M & L3-4 & - & Moderate \\
8 & 64 & M & L4-5 & + & Slight \\
9 & 64 & F & L4-5 & - & Slight \\
10 & 45 & M & L3-4 & - & Slight \\
11 & 78 & F & L4-5 & - & Marked \\
\hline
\end{tabular}


ness of the lower extremities was slightly improved in one of four patients who exhibited muscle weakness before examination. However, all of these patients had the recurrence of symptoms within a week after the examination and were treated surgically within 1 month after examination.

None of the patients who exhibited no morphological changes in the vessels along the cauda equina after the administration of PGE1 experienced any improvement of symptoms after examination. All of these patients were also treated surgically.

Results of surgery were evaluated by assessment of treatment of low back pain as proposed by the Japanese Orthopaedic Association (JOA score, full score: 29 points). ${ }^{15}$ Mean postoperative JOA score was 22 points for patients who exhibited dilation of vessels on the surface of the cauda equina, and 17 points for patients who exhibited no morphological changes in the vessels along the cauda equina. This difference was not found significant by the Mann-Whitney test.

As an adverse reaction, transient hypotension was observed in 8 of the 11 patients following PGE1 administration. However, the hypotension was mild (the range of decrease in mean blood pressure was $10 \mathrm{mmHg}$ to $20 \mathrm{mmHg}$ ). Only one patient felt sick. The hypotension was successfully treated with physiological saline alone, administered intravenously. No persistent cerebrospinal fluid leakage was observed, and no serious systemic complications were encountered in these patients.

\section{Case report}

A 51-year-old woman had experienced numbness and pain in the left leg for the previous 2 years. Recently, numbness had also developed in her right leg and she had difficulty in walking. Her myelogram revealed partial defects of contrast medium at the L4-5 and L5S1 levels (Figure 1a and b).

Myeloscopy was performed at the L4-5 level, and the vessels on the surface of the cauda equina were observed. Adhesions were evaluated as being of moderate degree. After an intravenous injection of Lipo PGE1, dilation of the blood vessels running on the cauda equina was observed (Figure 1c).

\section{Discussion}

For many years, there has been a great deal of debate surrounding the mechanism of development of symptoms of lumbar spinal canal stenosis. It has been thought that the mechanism of causation might not be limited to mechanical compression of the nerves by thickening ligaments, degenerated bones, or a protruded intervertebral disk, but that circulatory failure might be involved as well. In particular, significant involvement of blood circulation in the cauda equina and nerve root in the development of neurogenic intermittent claudication in this disease has been considered. Both a congestion theory based on circulatory failure of the veins in the cauda equina and nerve roots, and an ischemia theory based on decreased arterial blood flow have been proposed, but no agreement has been reached to date concerning which theory is correct. ${ }^{16}$

On behalf of the congestion theory, Kavanaugh et $a l^{4}$ have stated that stenosis of the spinal canal raises spinal fluid pressure in the caudal regions from the stenosed level, and when the elevated pressure exceeds the venous pressure in the cauda equina, venous perfusion failure results. Congestion in the cauda equina or nerve root is then induced, and anoxia develops. Ooi et $a l^{6}$ examined the caudia equina of patients with lumbar spinal canal stenosis myeloscopically, and reported that blood vessels on the cauda equina appeared to be dilated after the patients walked on a treadmill for a few minutes. They inferred that this vascular dilation was due to elevation of the capillary circulation pressure in the spinal canal caused by congestion in the epidural venous plexus.

In contrast, there are many findings in the literature supporting the ischemia theory. Olmarker et $a l^{5}$ have reported that compression applied to the pig's lumbar dura and cauda equina by an inflatable polyethylene cuff above $50 \mathrm{mmHg}$ progressively reduces the glucose supply to the constricted area. Because vasodilation has been observed in the ipsolateral spinal cord following exercise-loading with electrical stimuli on the unilateral hind limbs of mice, Blau and Rushworth ${ }^{2}$ believe that, in the presence of abnormalities in the spinal cord, anoxia is induced by exercise-loading on the inferior limbs because the blood circulation does not increase when it should in response to requirements of the natural metabolism of the spinal cord. On the assumption that the walking distance before the onset of claudicating pain is extended when partial oxygen pressure in the gas being breathed is raised, Evans ${ }^{3}$ has inferred that intermittent claudication can be attributed to the decreased oxygen supply to the cauda equina and nerve roots due to decreased blood circulation. Using electrophysiological methods, Tsuji et $a l^{7}$ have demonstrated the involvement of both static factors such as constriction of the neural tissue by a narrow spinal canal, and dynamic factors such as dynamic mechanical compression and an ischemic state in the cauda equina and nerve roots due to walking.

Previously, we compared myeloscopic findings with the surgical outcomes of patients with lumbar spinal canal stenosis, and reported that patients with marked adhesions had operative results distinctly inferior to those of patients with slight or moderate adhesions. ${ }^{13}$ Based on the results of the previous and current studies, we believe that decreased arterial blood flow produced by static and dynamic mechanical compression and adhesive arachnoiditis may be the main cause of intermittent claudication, although probably both venous and arterial failure are involved.

Doses of PGE1 are 60-95\% metabolized and inactivated in a single pulmonary circulation, and 
a

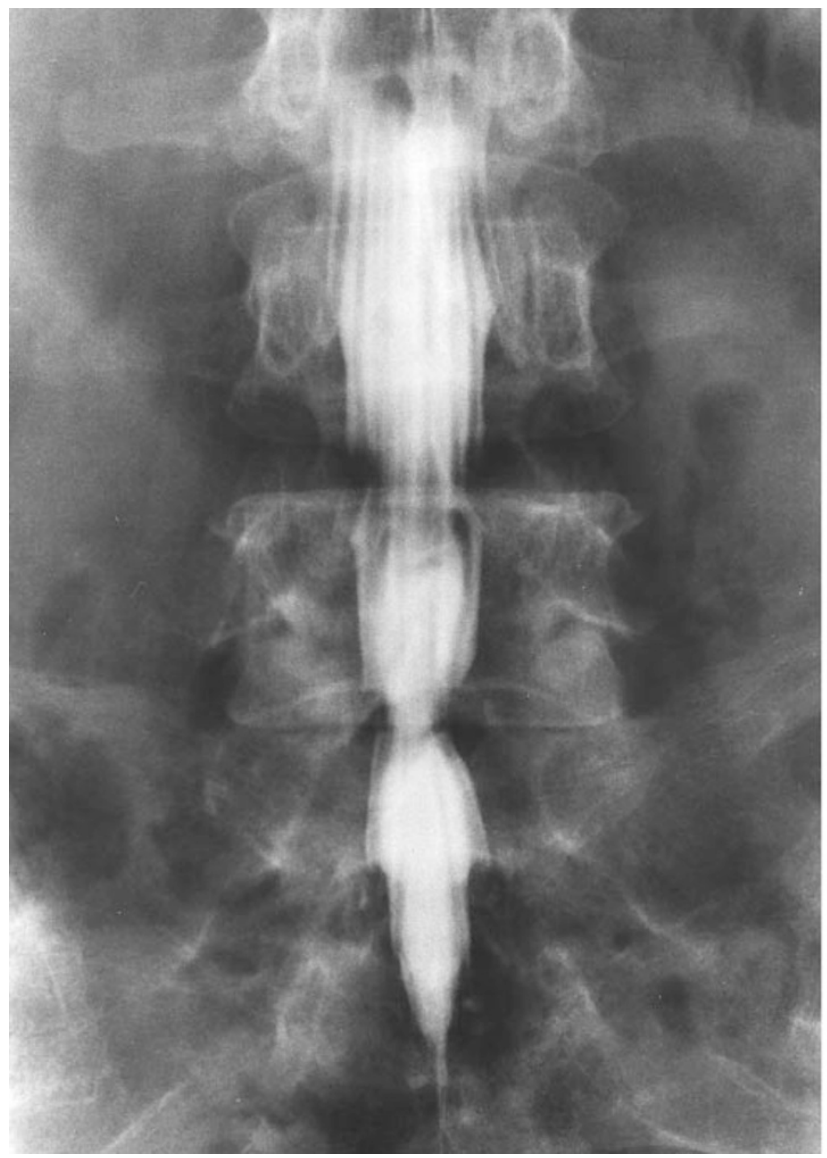

b

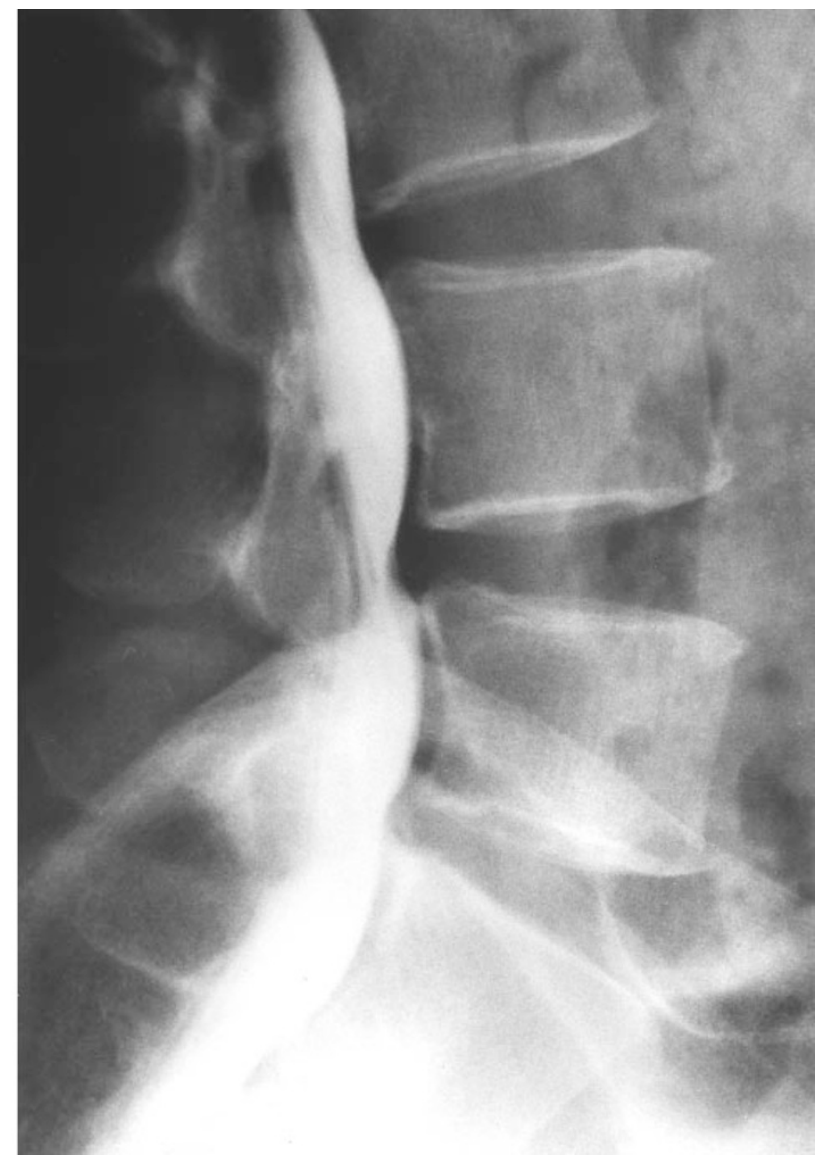

C

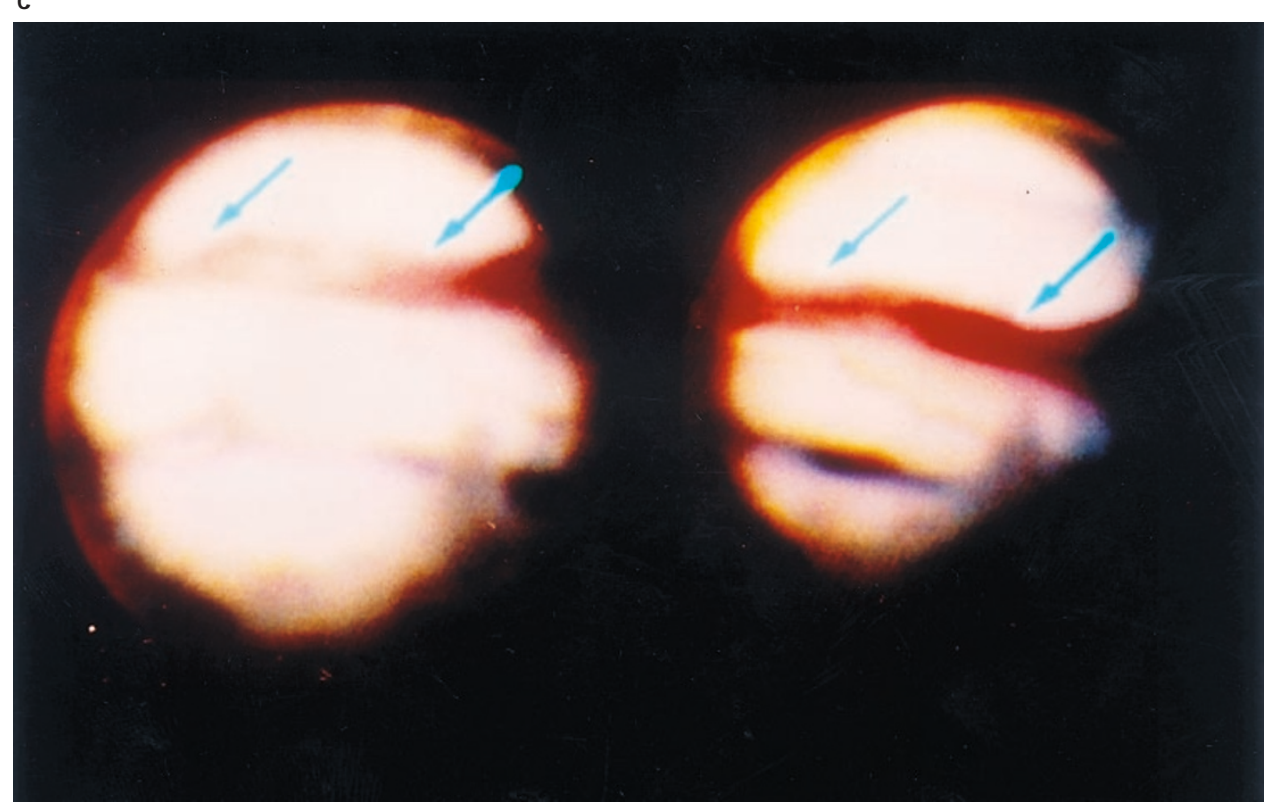

Figure 1 Case 1 a 51-year-old woman. (a) Anteroposterior view of myelogram. (b) Lateral view of myelogram. An incomplete block was observed at L4-5 and L5-S1 levels. (c) Myeloscopic findings at L4-L5 level. Left side: before administration of PGE1. Right side: after administration of PGE1. After intravenous injection of Lipo PGE1, dilation of blood vessels (arrow) running on the cauda equina was found 
therefore, a large dose is required for intravenous administration. ${ }^{17}$ Consequently, rubor at the injection site and vascular pain can occur as adverse reactions. In addition, fever, diarrhea, and hepatic dysfunction have been reported as systemic complications. ${ }^{14}$ In order to avoid these problems, PGE1 was mixed with microparticles $0.2 \mu \mathrm{m}$ in diameter made of soybean oil. Because PGE1 is incorporated into the lipid particles in this preparation, it is less susceptible to inactivation in the lung. In addition, this preparation characteristically becomes concentrated and acts selectively in lesions because the lipid particles adhere to the endothelial cells of injured blood vessels, and PGE1containing lipid particles are actively ingested by smooth muscle cells exposed to the bottom layer of the endothelium and macrophages. ${ }^{18,19}$ Therefore, sufficient clinical efficacy of this preparation can be expected with administration of a quarter to an eighth of the dose of conventional PGE1 preparations. Additional advantages of this preparation are that intravenous bolus injection is possible, and complications such as vascular pain are infrequent.

Ono et $a l^{10}$ have conducted a multi-institutional study of the clinical efficacy of Lipo PGE1 in 140 patients with lumbar spinal canal stenosis, and reported that although alleviation of lumbago was not obtained, significant improvement of intermittent claudication and numbness and pain in the lower extremities was achieved in about $40 \%$ of patients after 14 administrations of Lipo PGE1. Murakami et $a l^{9}$ treated 30 patients with lumbar spinal canal stenosis with ten administrations of Lipo PGE1, and achieved improvement in pain and numbness of the lower extremities, and intermittent claudication in 22 patients. They also reported that blood flow in the nerve root increased after the administration of Lipo PGE1 in measurements of blood flow during operation and in experimental study using cats. However, all of these measurements of blood flow were performed without compression of the caudia equina or nerve root, or after surgical decompression.

Using myeloscopy, we examined the effect of Lipo PGE1 on the cauda equina in patients with spinal canal stenosis. In the current series, 6 of 11 patients who underwent myeloscopic examination with administration of Lipo PGE1 exhibited dilation of the blood vessels on the surface of the cauda equina and experienced improvement of their symptoms. In our previous study, however, we performed myeloscopic examination without administration of PGE1 on 36 patients with lumbar spinal stenosis, and none exhibited dilation of the blood vessels on the surface of the cauda equina or improvement of symptoms after examination. ${ }^{13}$ These findings suggest that Lipo PGE1 may improve blood circulation in cauda equina, which probably improves clinical symptoms in some patients with lumbar spinal stenosis. In patients who experienced no improvement of clinical symptoms after administration of Lipo PGE1, the smooth muscle cells of the vascular walls may have been injured markedly, so that Lipo PGE1 may not improve blood circulation in the cauda equina or clinical symptoms.

In this study, all patients who experienced improvement of their symptoms had recurrence of symptoms within a week after examination. Murakami et al also reported that $62 \%$ of the patients whose symptoms improved after treatment with Lipo PGE1 experienced a recurrence of symptoms by 3 months after treatment. Thus, it is necessary to determine how many times or what dose of Lipo PGE1 administration is required to maintain improved clinical symptoms.

In conclusion, the findings of this study suggest that Lipo PGE1 may enhance blood flow in the cauda equina and improve clinical symptoms in some patients with lumbar spinal stenosis. However, the number of patients in this study was small and the range of observation by myeloscopy was restricted. Further study is needed to clarify the effect of PGE1 on the cauda equina in patients with lumbar spinal stenosis.

\section{References}

1 Verbiest H. A radicular syndrome from developmental narrowing of the lumbar vertebral canal. J Bone Joint Surg 1954; 36B: $230-237$.

2 Blau JN, Rushworth G. Observation on the blood vessels of the spinal cord and their responses to motor activity. Brain 1958; 81: $354-363$

3 Evans JG. Neurogenic intermittent claudication. $\mathrm{Br}$ Med J 1964; 2: $985-987$.

4 Kavanaugh GJ, Svien HJ, Holman CB, Johnson RM. 'Pseudoclaudication' syndrome produced by compression of the cauda equina. JAMA 1968; 206: 2477-2481.

5 Olmarker K, Rydevik B, Holm S. Edema formation in spinal nerve roots induced by experimental, graded compression. Spine 1989; 14: $569-573$

6 Ooi Y, Mita F, Satoh Y. Myeloscopic study on lumbar spinal canal stenosis with special reference to intermittent claudication. Spine 1990; 15: $544-549$.

7 Tsuji $\mathrm{H}$ et al. Redundant nerve root in patients with degenerative lumbar spinal stenosis. Spine 1985; 10: $72-82$.

8 Wilson CB. Significance of the small lumbar spinal canal: cauda equina compression syndrome due to spondylosis. Part 3: intermittent claudication. J Neurosurg 1969; 31: 499-506.

9 Murakami M et al. Effects of intravenous administration of Lipo PGE1 for lumbar spinal canal stenosis. Rinsho Seikei Geka 1992; 27: $1011-1018$ (in Japanese).

10 Ono K, Miura S, Tuji H, Kataoka O. Clinical assessment of PGE1 formation for lumbar spinal canal stenosis. Spin \& Spinal Cord 1997; 10: 517 - 524 (in Japanese).

11 Clifford PC et al. Treatment of vasospastic disease with prostaglandin $\mathrm{E}_{1}$. Br Med J 1980; 281: 1031 - 1034.

12 Martin MFR et al. Prostaglandin $\mathrm{E}_{1}$ infusions for vascular insufficiency in progressive systemic sclerosis. Ann Rheum Dis 1981; 40: $350-354$.

13 Kawauchi Y, Yone K, Sakou T. Myeloscopic observation of adhesive arachnoiditis in patients with lumbar spinal stenosis. Spinal Cord 1996; 34: 403-410.

14 Mizushima Y, Yanagawa A, Hoshi K. Prostaglandin E1 is more effective, when incorporated in lipid microsheres, for treatment of peripheral vascular diseases in man. J Pharm Pharmacol 1983; 35: $667-668$.

15 Japanese Orthopaedic Association Committe. Assessment of treatment of low back pain. J Jpn Orthop Ass 1984; 58: 952 - 955. 
16 Edgar MA. The pathogenesis of low back pain and leg pain. In: Nixon JE (ed). Spinal stenosis. Edward Arnold: London 1991, pp 36-54.

17 Hammond GL, Cronau LH, Whittaker D, Gillis CN. Fate of prostaglandins $\mathrm{E}_{1}$ and $\mathrm{A}_{1}$ in the human pulmonary circulation. Surgery 1977; 81: $716-722$.
18 Hallberg D. Elimination of exogenous lipids from the blood stream. Acta Physiol 1965; 254: 1-23.

19 Mizushima Y, Yanagawa T, Yokoyama K. Tissue distribution and anti-inflammatory activity of corticosteroids incorporated in lipid emulsion. Ann Rheum Dis 1982; 41: 263-267. 